\title{
No-waiting segmentectomy: an optimized approach for segmentectomy
}

\author{
Changchun Wang ${ }^{1,2 \#}$, Lei Cai ${ }^{1,2 \#}$, Qian Chen ${ }^{1,2}$, Xiaofang $\mathbf{X u}^{1,2}$, Jinxiao Liang ${ }^{1,2}$, Weimin Mao ${ }^{1,2,3 *}$, \\ Qixun Chen ${ }^{1,2,3 *}$
}

${ }^{1}$ Department of Thoracic Surgery, Cancer Hospital of the University of Chinese Academy of Sciences (Zhejiang Cancer Hospital), Hangzhou, China; ${ }^{2}$ Institute of Cancer and Basic Medicine (ICBM), Chinese Academy of Sciences, Hangzhou, China; ${ }^{3}$ Department of Thoracic Surgery, Zhejiang Key Laboratory of Diagnosis and Treatment Technology on Thoracic Oncology, Zhejiang Cancer Hospital, Hangzhou, China

Contributions: (I) Conception and design: Q Chen; (II) Administrative support: W Mao; (III) Provision of study materials or patients: Q Chen, X Xu, J Liang; (IV) Collection and assembly of data: L Cai, C Wang; (V) Data analysis and interpretation: C Wang; (VI) Manuscript writing: All authors; (VII) Final approval of manuscript: All authors.

\#These authors contributed equally to this work.

*These authors contributed equally to this work.

Correspondence to: Qixun Chen, MD; Weimin Mao, MD. Department of Thoracic Surgery, Cancer Hospital of the University of Chinese Academy of Sciences (Zhejiang Cancer Hospital), Hangzhou 310022, China; Institute of Cancer and Basic Medicine (ICBM), Chinese Academy of Sciences, Hangzhou 310022, China; Department of Thoracic Surgery, Zhejiang Key Laboratory of Diagnosis and Treatment Technology on Thoracic Oncology, Zhejiang Cancer Hospital, Hangzhou 310022, China. Email: chenqixun64@163.com; maowm1218@163.com.

Background: Currently, modified inflation-deflation is considered the easiest way to identify the intersegmental plane during pulmonary segmentectomy. However, this approach requires a wait of about 1020 min during the operative procedure. Therefore, we optimized the procedure, which we call no-waiting segmentectomy. In this study, we compared no-waiting segmentectomy with the modified inflation-deflation method.

Methods: We studied 123 consecutive patients with pulmonary ground-glass nodules who underwent segmentectomy by uniportal video-assisted thoracoscopic surgery in a single medical group from January 2019 to April 2020. Forty-five patients underwent the modified inflation-deflation method and 78 patients underwent the no-waiting method. The no-waiting procedure involved severing of the target segmental pulmonary artery, inflating the lung with atmospheric air, dissecting the hilum, and dividing the target segmental bronchus. The entire procedure could be performed at a stretch and no pause was needed. We compared the two methods for surgery time, bleeding volume, drainage time, and postoperative hospital stay. Propensity-score matching was used to adjust the baseline characteristics.

Results: Thirty-three pairs of 123 patients were successfully matched. Before propensity-score matching, there was no difference between the two methods in terms of surgery time, bleeding volume, drainage time, and postoperative hospital stay. After propensity-score matching, the surgery time in the no-waiting group was significantly shorter than that in the modified inflation-deflation method group $(80.12 \pm 35.53 \mathrm{vs}$. $102.97 \pm 48.07 \mathrm{~min}, \mathrm{P}=0.03)$. There was no difference between the two methods in terms of bleeding volume, drainage time, and postoperative hospital stay.

Conclusions: No-waiting segmentectomy was associated with a reduced surgery time, compared to that associated with modified inflation-deflation segmentectomy. Furthermore, no-waiting segmentectomy did not increase bleeding volume, drainage time, and postoperative hospital stay. Thus, no-waiting segmentectomy is an optional optimized approach for segmentectomy.

Keywords: Pulmonary segmentectomy; no-waiting; modified inflation-deflation method; intersegmental planes

Submitted Aug 14, 2020. Accepted for publication Dec 04, 2020.

doi: $10.21037 /$ jtd-20-2661

View this article at: http://dx.doi.org/10.21037/jtd-20-2661

(C) Journal of Thoracic Disease. All rights reserved. 


\section{Introduction}

There has been a marked increase in the number of small lung nodules and nonsolid lung cancers being identified because of the advances in computed tomography (CT) technology over the last decade. The National Comprehensive Cancer Network guidelines recommend segmentectomy for early-stage lung cancer, but the determination of the intersegmental planes can be difficult. There are two types of methods (1-8). One type determines the intersegmental plane through bronchi. Surgeons use selective jet ventilation of the target segmental bronchus (1) and inject a stain into the bronchus $(4,5,7)$. The other type depends on vessels. Surgeons inject indocyanine green through the central vein or the target pulmonary arteries (9). Both methods require special equipment or materials. Chen Liang has described a modified inflationdeflation approach (3) that does not require special equipment or materials; however, it requires a waiting period of 10-20 min after inflation. To avoid this pause in the surgery procedure, we recently developed an optimized approach, which we call "no-waiting segmentectomy". In this method, the following procedures are performed in sequence to reveal the intersegmental plane: identification and cutting of the target segmental arteries, inflation of the lung, dissection of the hilar structures, and cutting of the target segmental bronchus. The process does not require special equipment and materials or a waiting period. We believe that this is the easiest way to identify the intersegmental plane. In this study, we describe this method and retrospectively compare it with the modified inflationdeflation approach. We present the following article in accordance with the STROBE reporting checklist (available at http://dx.doi.org/10.21037/jtd-20-2661).

\section{Methods}

The study was conducted in accordance with the Declaration of Helsinki (as revised in 2013). The ethics committee of Zhejiang Cancer Hospital approved this retrospective study (IRB-2019-188) and waived the need for individual consent.

\section{Patients}

A total of 123 patients with ground-glass nodules underwent segmentectomy by uniportal video-assisted thoracoscopic surgery (UVATS) in a single medical group between
January 2019 and March 2020. Of them, 45 underwent the modified inflation-deflation approach, while 78 underwent no-waiting segmentectomy. The former and the latter were considered as the control group and the study group, respectively. Lung function parameters were obtained from lung functional reports. Emphysema was diagnosed according to CT reports. Thoracic adhesion and surgical procedure times were recorded according to surgical records.

\section{No-waiting segmentectomy procedure}

All patients underwent 1-mm thin-layer CT; DICOM format data were used for three-dimensional (3D) reconstruction. For the surgery, the patient was placed in the lateral position, and the lung was isolated with a doublelumen endotracheal tube. Using the UVATS approach, a 3 -cm-long incision is made in the posterior axillary line at the upper edge of the fifth rib. The target segmental pulmonary artery is identified and severed according to the 3D image. Then, the lungs are inflated with atmospheric air. Without waiting for the lungs to collapse, the hilum is revealed using an oval clamp and the hilar nodes are dissected; then, the target segmental bronchus is divided. On deflation of the lung, the segments to be preserved collapse and lung parenchyma turns dark purple, while the target segment collapses but remains pink. A demarcation line appears between the target segment and the lung that is to be preserved; this represents the anatomic intersegmental plane. The duration from inflation of the lungs and the appearance of the intersegmental plane is usually 10-15 min. The target segment bronchus is severed, and the target segment resected using an ultrasonic knife and stapler. The target segmental bronchus can be cut either immediately after the lung is inflated or after the lymph nodes are removed. After it is cut, the distal stump can be closed or left open; this does not affect the formation of the intersegmental plane (Video 1).

The dissection of the segmental vein is minimized throughout the procedure. The intrasegmental vein is cut when the intraoperative pulmonary veins are consistent with the preoperative $3 \mathrm{D}$ reconstruction image; otherwise, the veins are cut as less as possible. The intrasegmental veins in the upper lungs are usually cut before inflation of the lungs because the veins are located at the surface of the hilum; however, intrasegmental veins in the lower lungs are situated deeper in the lung tissues. Lifting the distal stump of the target segmental bronchus and cutting only the veins 
Table 1 Baseline characteristics of patients before and after propensity score matching

\begin{tabular}{|c|c|c|c|c|c|c|}
\hline Characteristics & \multicolumn{3}{|c|}{ Before propensity score matching } & \multicolumn{3}{|c|}{ After propensity score matching } \\
\hline Age (mean $\pm S D$ ), years & $54.22 \pm 12.39$ & $53.22 \pm 12.00$ & 0.66 & $54.12 \pm 12.11$ & $50.06 \pm 11.79$ & 0.17 \\
\hline Location & $5.29 \pm 3.56$ & $6.81 \pm 4.37$ & 0.04 & $5.94 \pm 3.47$ & $5.27 \pm 3.03$ & 0.41 \\
\hline FEV1 (mean \pm SD) & $2.36 \pm 0.71$ & $2.44 \pm 0.63$ & 0.53 & $2.34 \pm 0.65$ & $2.50 \pm 0.66$ & 0.33 \\
\hline FEV1\% (mean \pm SD) & $85.80 \pm 18.83$ & $87.52 \pm 13.56$ & 0.56 & $85.45 \pm 16.74$ & $89.58 \pm 12.72$ & 0.26 \\
\hline FEV1/FVC (mean \pm SD) & $104.91 \pm 15.07$ & $103.95 \pm 6.57$ & 0.69 & $104.55 \pm 13.93$ & $105.85 \pm 5.17$ & 0.62 \\
\hline
\end{tabular}

that go to the target segment can simplify the procedure.

\section{Modified inflation-deflation segmentectomy}

In the modified inflation-deflation segmentectomy, the target segmental bronchus, arteries, and veins are cut and both lungs are inflated with pure oxygen; the lungs are then deflated naturally, and the operation is paused. The target segment is identified after an interval of 10-20 min.

\section{Statistical analysis}

Continuous data were reported as mean \pm standard deviation and variables between treatment groups were compared using an independent-sample $t$-test. Categorical data were reported as numbers with percentages and Pearson's Chisquare test was used to compare the differences. To adjust for the imbalances in baseline characteristics in different treatment groups, propensity-score matching (PSM) was performed using one-to-one nearest-neighbor matching within a caliper of 0.02 . No replacement was allowed. To meet PSM requirements, we randomly assigned the location of a different segment to a different number. We created a propensity score for the no-waiting segmentectomy cohort by using logistic regression based on potential confounding variables, including age, gender, tumor location, thoracic adhesion, emphysema, lung functional parameters (FVC, FVC\%, FEV1, FEV1\%, FEV1/FVC). All statistical analyses were two-sided and $\mathrm{P}<0.05$ was considered statistically significant. The analyses were performed using SPSS 23.0 (IBM Corp., Armonk, NY, USA).

\section{Results}

A total of 123 patients who received segmentectomy (modified inflation-deflation, $45 v s$. no waiting, 78) were included in this study. The baseline characteristics of patients are summarized in Table 1. In the unmatched cohort, the locations of segments differed between the two groups, but the other baseline characteristics such as age, sex, thoracic adhesion, emphysema and lung function parameters were not significantly different. PSM yielded a cohort of 66 patients that was well-balanced on all preoperative characteristics (Table 1).

Before PSM, there were no differences between the two groups in terms of surgery time, bleeding volume, drainage time and hospital days. After PSM, the surgery time in the no-waiting group was significantly shorter than that in the modified inflation-deflation group $(80.12 \pm 35.53$ vs. $102.97 \pm 48.07, \mathrm{P}=0.03)$. However, bleeding volume, drainage time, and hospital days were not different between the two groups (Table 2).

\section{Discussion}

In this study, we compared two methods of lung segment plane display through PSM and found that no waiting shortened the operation time, but did not increase 
Table 2 Operative results of patients before and after propensity score matching

\begin{tabular}{|c|c|c|c|c|c|c|}
\hline Results & \multicolumn{3}{|c|}{ Before propensity score matching } & \multicolumn{3}{|c|}{ After propensity score matching } \\
\hline Operating time (min) & $103.89 \pm 44.30$ & $100.65 \pm 44.48$ & 0.69 & $102.97 \pm 48.07$ & $80.12 \pm 35.53$ & 0.03 \\
\hline Bleeding volume (mL) & $72.00 \pm 82.04$ & $66.92 \pm 67.25$ & 0.71 & $74.24 \pm 91.07$ & $52.12 \pm 48.85$ & 0.23 \\
\hline Drainage time (days) & $2.89 \pm 3.51$ & $2.38 \pm 1.46$ & 0.36 & $3.0 \pm 4.02$ & $2.51 \pm 1.72$ & 0.48 \\
\hline
\end{tabular}

complications. This implies that no waiting optimizes the surgical procedure and is an alternative and optimized method for identifying the plane of the lung segment.

The patients in this study were from the same medical group, thus avoiding operator bias. In addition, all patients were continuously enrolled, thus avoiding selection bias. The segmentectomies were performed with the modified inflation-deflation method before August 2019 and with no waiting after August 2019. The two chief surgeons in this medical group have more than 20 years of surgical experience and their surgical level did not change significantly over the study period. On the other hand, all our operations were UVATS. Because of the uniportal perspective, the hilum can be clearly revealed even if the lung is not completely collapsed.

Since it can be performed without special equipment or materials, the modified inflation-deflation method is a widely used method in segmentectomy, especially in China. There are some differences between modified inflationdeflation method and no waiting. In the modified inflationdeflation method, both the target segmental bronchus and vessels are severed first, and high pressure $\left(20 \mathrm{cmH}_{2} \mathrm{O}\right)$ is applied to fully inflate the lung including the target segment, after which the operation is suspended for 5$12 \mathrm{~min}$ (3). In no-waiting segmentectomy, a slight pressure can fully inflate the entire lung; importantly, there is no need to pause the operation. We advanced this period of pause and waiting in the modified inflation-deflation method to perform lymph node resection and bronchi dissection in no-waiting segmentectomy.

In the no-waiting group, the complete intersegmental plane appeared after the target segmental arteries were cut, regardless of whether the distal stumps of the target segmental bronchi were closed. Thus, in this method, accurate cutting of the target pulmonary arteries appears to be the decisive factor in the formation of the intersegmental plane. Zhao et al. reported a similar approach (10).

Intersegmental veins are the anatomical marks of lung segments. In the no-waiting method, the intersegmental veins can be revealed between the pink and purple parts when the demarcation line is divided with an ultrasonic knife, which indicates the intersegmental planes are accurate (Video 2). To obtain sufficient margins, joint segment or joint subsegment resection is performed if the lesions are near the intersegmental planes; the decision can be made using $3 \mathrm{D}$ reconstruction before the operation. As shown in Video 2, the margin ball was $2 \mathrm{~cm}$ away from the lesion margin.

A possible mechanism underlying the formation of the intersegmental plane in no-waiting segmentectomy could be as follows (11): after the target segmental arteries are cut and the lungs are inflated, both the target segment and the segments to be preserved are ventilated and appear pink. When the lung is deflated gradually, the segments to be preserved continue to be perfused with pulmonary arterial blood (deoxygenated blood) and absorb oxygen. Meanwhile, in the target segment, the alveoli cannot absorb oxygen due to the absence of pulmonary circulation. The time taken for the preserved segments to turn from pink to purple is about 10-20 minutes.

To ensure accurate cutting of the target segmental arteries, preoperative $3 \mathrm{D}$ reconstruction is usually necessary. In cases with a poor interlobular fissure, no waiting is not recommended for resection of S9, S10, or LS4+5 since the arteries are difficult to reveal. However, no waiting is appropriate in cases with good interlobular fissure for these segments.

There are some limitations of this retrospective study. We observed that in both methods, accurate severing of the target artery was the key to the formation of the intersegmental plane; the bronchi appear to have no role. The explanation that we have proposed is only based on 
conjecture. Almost all authors inflate the lungs with pure oxygen, while we inflated the lungs with atmospheric air and obtained the same results. We do not know the exact logic underlying the formation of the intersegmental plane.

\section{Acknowledgments}

We would like to acknowledge Wen Cheng, a software engineer, for his $3 \mathrm{D}$ reconstruction.

Funding: This study was supported by the Zhejiang Provincial Natural Science - Mathematical Medicine Association Joint Fund (LSY19H160001) and the Medical Science and Technology Project of Zhejiang Province(2013KYA031, 2021KY560).

\section{Footnote}

Reporting Checklist: The authors have completed the STROBE reporting checklist. Available at http://dx.doi. org/10.21037/jtd-20-2661

Data Sharing Statement: Available at http://dx.doi. org/10.21037/jtd-20-2661

Peer Review File: Available at http://dx.doi.org/10.21037/jtd20-2661

Conflicts of Interest: All authors have completed the ICMJE uniform disclosure form (available at http://dx.doi. org/10.21037/jtd-20-2661). The authors have no conflicts of interest to declare.

Ethical Statement: The authors are accountable for all aspects of the work in ensuring that questions related to the accuracy or integrity of any part of the work are appropriately investigated and resolved. The study was conducted in accordance with the Declaration of Helsinki (as revised in 2013). The ethics committee of Zhejiang Cancer Hospital approved this retrospective study (IRB-2019-188) and waived the need for individual consent.

Open Access Statement: This is an Open Access article distributed in accordance with the Creative Commons Attribution-NonCommercial-NoDerivs 4.0 International License (CC BY-NC-ND 4.0), which permits the noncommercial replication and distribution of the article with the strict proviso that no changes or edits are made and the original work is properly cited (including links to both the formal publication through the relevant DOI and the license). See: https://creativecommons.org/licenses/by-nc-nd/4.0/.

\section{References}

1. Okada M, Mimura T, Ikegaki J, et al. A novel video-assisted anatomic segmentectomy technique: selective segmental inflation via bronchofiberoptic jet followed by cautery cutting. J Thorac Cardiovasc Surg 2007;133:753-8.

2. Misaki N, Chang SS, Igai H, et al. New clinically applicable method for visualizing adjacent lung segments using an infrared thoracoscopy system. J Thorac Cardiovasc Surg 2010;140:752-6.

3. Wang J, Xu X, Wen W, et al. Modified method for distinguishing the intersegmental border for lung segmentectomy. Thorac Cancer 2018;9:330-3.

4. Sekine $\mathrm{Y}, \mathrm{Ko} \mathrm{E}$, Oishi $\mathrm{H}$, et al. A simple and effective technique for identification of intersegmental planes by infrared thoracoscopy after transbronchial injection of indocyanine green. J Thorac Cardiovasc Surg 2012;143:1330-5.

5. Zhang Z, Liao Y, Ai B, et al. Methylene blue staining: a new technique for identifying intersegmental planes in anatomic segmentectomy. Ann Thorac Surg 2015;99:238-42.

6. Kasai Y, Yokomise H. Infrared thoracoscopy. Kyobu Geka 2014;67:758-63.

7. Oh S, Suzuki K, Miyasaka Y, et al. New technique for lung segmentectomy using indocyanine green injection. Ann Thorac Surg 2013;95:2188-90.

8. Kim DY, Jeong JY. Robotic surgery for pulmonary segmentectomy. J Thorac Dis 2019;11:624-7.

9. Pischik VG, Kovalenko A. The role of indocyanine green fluorescence for intersegmental plane identification during video-assisted thoracoscopic surgery segmentectomies. J Thorac Dis 2018;10:S3704-11.

10. Zhao Y, Xuan Y, Song J, et al. A novel technique for identification of the segments based on pulmonary artery plane combined with oxygen diffusing discrepancy. J Thorac Dis 2019;11:5427-32.

11. Iwata H, Shirahashi K, Mizuno Y, et al. Surgical technique of lung segmental resection with two intersegmental planes. Interact Cardiovasc Thorac Surg 2013;16:423-5.

Cite this article as: Wang C, Cai L, Chen Q, Xu X, Liang J, Mao W, Chen Q. No-waiting segmentectomy: an optimized approach for segmentectomy. J Thorac Dis 2021;13(2):784-788. doi: $10.21037 /$ jtd-20-2661 